\title{
PhotoATRP Induced Self-Assembly (PhotoATR-PISA) Enables Simplified Synthesis of Responsive Polymer Nanoparticles in One-Pot
}

\author{
Ali Shahrokhinia ${ }^{a}$, Randall A. Scanga ${ }^{a}$, Priyanka Biswas ${ }^{a}$, James F. Reuther ${ }^{\text {** }}$ \\ ${ }^{a}$ Department of Chemistry, University of Massachusetts Lowell, Lowell, MA
}

\section{Supporting information}

Table of Contents:

1. Small molecule synthesis

1.1. Synthesis of furan-based initiator

1.2. Synthesis of N,N-Cystaminebismethacrylamide (CBMA) crosslinker

2. Characterization methods

2.1. Gel permeation chromatography (GPC)

2.2. NMR spectroscopy

2.3. Dynamic light scattering (DLS)

2.4. Atomic force microscopy (AFM)

2.5 Transmission Electron Microscopy (TEM)

2.6. Fluorescence spectroscopy

3. Supporting Figures and Tables

4. References 


\section{Small molecule synthesis}

\subsection{Synthesis of furan-based initiator}

Furfuryl alcohol (1.0 eq, $13.9 \mathrm{ml})$ and triethylamine (1.01 eq, $22.6 \mathrm{ml})$ were dissolved in $300 \mathrm{ml}$ dichloromethane (DCM) and cooled to $0{ }^{\circ} \mathrm{C}$. 2-bromoisobutyryl bromide (1.01 eq, $\left.20.0 \mathrm{ml}\right)$ was then added dropwise to the solution by an addition funnel. The reaction temperature was maintained at $0 \square \mathrm{C}$ for two hours and then gradually increased to the ambient temperature, after that the reaction continued for $22 \mathrm{hrs}$. The resulting brown solution was concentrated and subsequently washed with $1 \mathrm{M} \mathrm{HCl}(3 \times 100 \mathrm{ml}), 1 \mathrm{M} \mathrm{NaHCO}_{3}(3 \times 100 \mathrm{ml})$ and brine solution $(3 \times 100 \mathrm{ml})$. The organic layer was collected and dried over sodium sulfate, filtered, and the solvent was removed via rotatory evaporation. The crude residue was then subjected to flash column chromatography $\left(1: 9(\mathrm{v}: \mathrm{v})\right.$ EtOAc/n-hexane, $\left.\mathrm{R}_{\mathrm{f}}=0.5\right)$ to obtain the product as a dark yellow viscous liquid (yield $=76 \%$ ).

${ }^{1} \mathrm{H}-\mathrm{NMR}(400 \mathrm{MHz}, \mathrm{CHLOROFORM-D)} \delta 7.39$ (q, J = 0.8 Hz, 1H), 6.32-6.42 (m, 2H), 5.11 (s, 2H), $1.89(\mathrm{~s}, 6 \mathrm{H})$.

${ }^{13} \mathrm{C}-\mathrm{NMR}$ (101 MHz, CHLOROFORM-D) $\delta$ 171.32, 148.86, 143.51, 111.11, 110.69, 77.55, 77.23, $76.92,59.55,55.69,30.76$. 

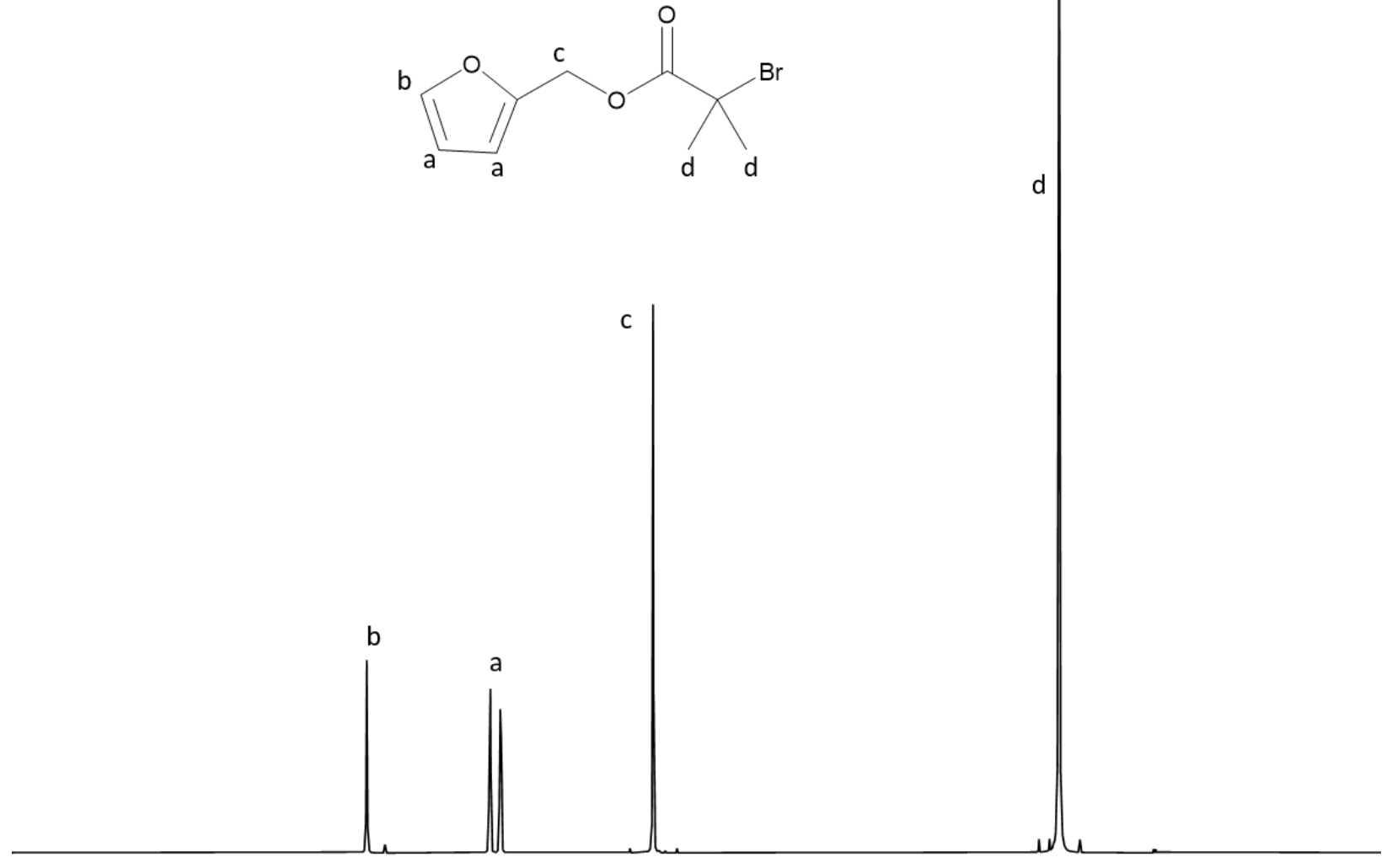

$\begin{array}{llllllllllllllllllllll}10.0 & 9.5 & 9.0 & 8.5 & 8.0 & 7.5 & 7.0 & 6.5 & 6.0 & 5.5 & 5.0 & 4.5 & 4.0 & 3.5 & 3.0 & 2.5 & 2.0 & 1.5 & 1.0 & 0.5 & 0.0 & -0.5 \\ \mathrm{f} 1(\mathrm{ppm}) & & & & & & \end{array}$

Figure S1. HNMR of furan-based initiator utilized to study PhotoATR-PISA

\subsection{Synthesis of $\mathrm{N}, \mathrm{N}-\mathrm{Cystaminebismethacrylamide} \mathrm{(CBMA)} \mathrm{crosslinker}$}

The synthesis of CBMA was adapted from previously reported literature protocols. ${ }^{1}$ Cystamine dihydrochloride (5.8 g, $25.7 \mathrm{mmol})$ and sodium hydroxide $(3.2 \mathrm{~g}, 80 \mathrm{mmol})$ were added into a 250 $\mathrm{mL}$ round bottomed flask containing $60 \mathrm{~mL}$ of water. Then, the flask was cooled to $0{ }^{\circ} \mathrm{C}$ in an icewater bath. After gently stirring for $30 \mathrm{~min}$, a solution of methacryloyl chloride (5.2 g, $49.7 \mathrm{mmol})$ 
in $20 \mathrm{~mL}$ of dichloromethane was added dropwise into the flask over 30 min via addition funnel resulting in formation white precipitate. After stirring for another $3 \mathrm{~h}$, the reaction mixture was filtered to collect the white precipitate. The synthesized solid was washed with deionized water three times and the crude product was recrystallized from ethyl acetate three times to obtain the resultant CBMA. ${ }^{1} \mathrm{H}$ NMR analysis conformed with previously reported spectra (Figure S2).

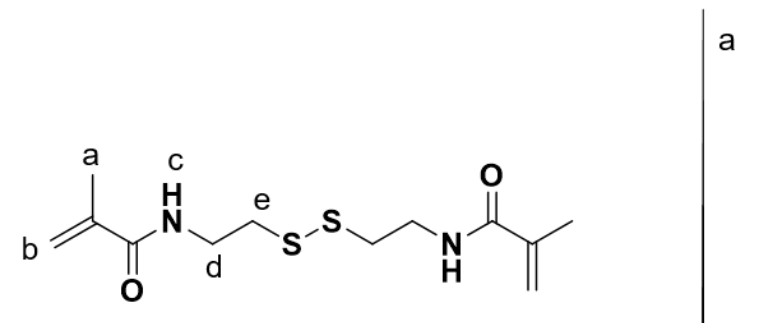

$\begin{array}{llllllllllllllllllllllllllllll}12.0 & 11.5 & 11.0 & 10.5 & 10.0 & 9.5 & 9.0 & 8.5 & 8.0 & 7.5 & 7.0 & 6.5 & 6.0 & 5.5 & 5.0 & 4.5 & 4.0 & 3.5 & 3.0 & 2.5 & 2.0 & 1.5 & 1.0 & 0.5 & 0.0 & -0.5 & -1.0 & -1.5 & -2.0\end{array}$

Figure S2. ${ }^{1} \mathrm{H}$ NMR of CBMA crosslinker after purification.

\section{Characterization methods}

\subsection{Gel permeation chromatography (GPC)}

Molecular weight $\left(\mathrm{M}_{\mathrm{n}}\right)$ and molecular weight distribution $\left(\mathrm{M}_{\mathrm{w}} / \mathrm{M}_{\mathrm{n}}\right)$ were measured using an HLC8320 EcoSEC GPC gel permeation chromatography (GPC), equipped with RI detector (set at 40 
$\left.{ }^{\circ} \mathrm{C}\right)$. Two ultra styragel columns were utilized in series and calibrated using monodispersed polystyrene standards and tetrahydrofuran (THF) as the eluent at a flow rate of $0.5 \mathrm{~mL} / \mathrm{min}$.

\subsection{NMR spectroscopy}

${ }^{1} \mathrm{H}$ and ${ }^{13} \mathrm{C}$ NMR spectra were recorded using a JEOL ECZ $400 \mathrm{MHz}$ spectrometer in $\mathrm{CDCl}_{3}$ and DMSO- $\mathrm{d}_{6}$ (Sigma-Aldrich) and referenced to residual solvent peaks. ${ }^{1} \mathrm{H}$ NMR spectra were collected averaging 16 scans. For kinetic studies, $10 \mathrm{~mol} \%$ of DMF was added to the reaction mixture as an internal standard to monitor monomer consumption over time.

\subsection{Dynamic light scattering (DLS)}

Dynamic light scattering (DLS) was carried using a Horiba SZ-100 Particle Analyzer to measure average hydrodynamic diameters for nanoparticles and their size distribution. The scattered light was detected at an angle of $173^{\circ}$. The samples were prepared by dissolving copolymers in filtered methanol with a concentration of $5.0 \mathrm{mg} / \mathrm{mL}$ at $25{ }^{\circ} \mathrm{C}$. The data processing was done using the general-purpose algorithms provided in the SZ-100 Software. Sample measurements were obtained in triple runs and reported as an average.

\subsection{Atomic force microscopy (AFM)}

AFM was performed using a Park Systems XE-100. Non-contact AFM mode was utilized to map the topography relying on an oscillating tip. The measurements were achieved using commercial cantilevers with a nominal spring constant and resonance frequency equivalent to $10-130 \mathrm{~N} / \mathrm{m}$ and 204-497 kHz, respectively (Park systems, PPP-NCHR). AFM samples were prepared by spincoating of the nanoparticle solution (diluted to $0.5 \mathrm{mg} / \mathrm{ml}$ ) on the bare mica disks (Diameter $=20$ $\mathrm{mm}$; Ted Pella) in the 4000 RPM for $1 \mathrm{~min}$. 


\subsection{Transmission Electron Microscopy (TEM)}

Transmission electron microscopy (TEM) was implemented utilizing a Philips CM12 electron microscope operated at $120 \mathrm{kV}$. The polymerization dispersions prepared at $65^{\circ} \mathrm{C}$ in different time intervals and at room temperature after dilution with methanol (1 $\mathrm{mg} / \mathrm{ml}$ dispersions $)$ were dropcast on carbon-coated copper grids and subsequently dried in a vacuum oven. The TEM imaging of all samples was done without any external staining.

\subsection{Fluorescence spectroscopy}

Fluorescence spectra for Nile Red encapsulated CCL NP samples were recorded on a JASCO FP8500 fluorescence spectrophotometer at the excitation and emission wavelengths of 540 and 630 $\mathrm{nm}$, respectively $(\mathrm{c}=5.0 \mathrm{mg} / \mathrm{ml}$ and at ambient temperature $)$.

\section{Supporting Figures and Tables}

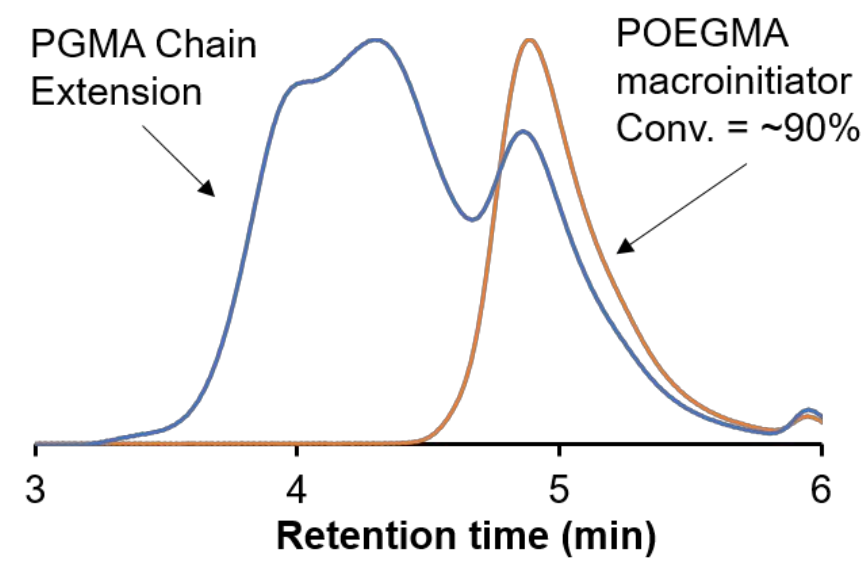

Figure S3. GPC chromatogram of attempted one-pot PhotoATR-PISA upon PGMA chain extension from POEGMA macroinitiators at $\sim 90 \%$ OEGMA monomer conversion showing poor reinitiation. 


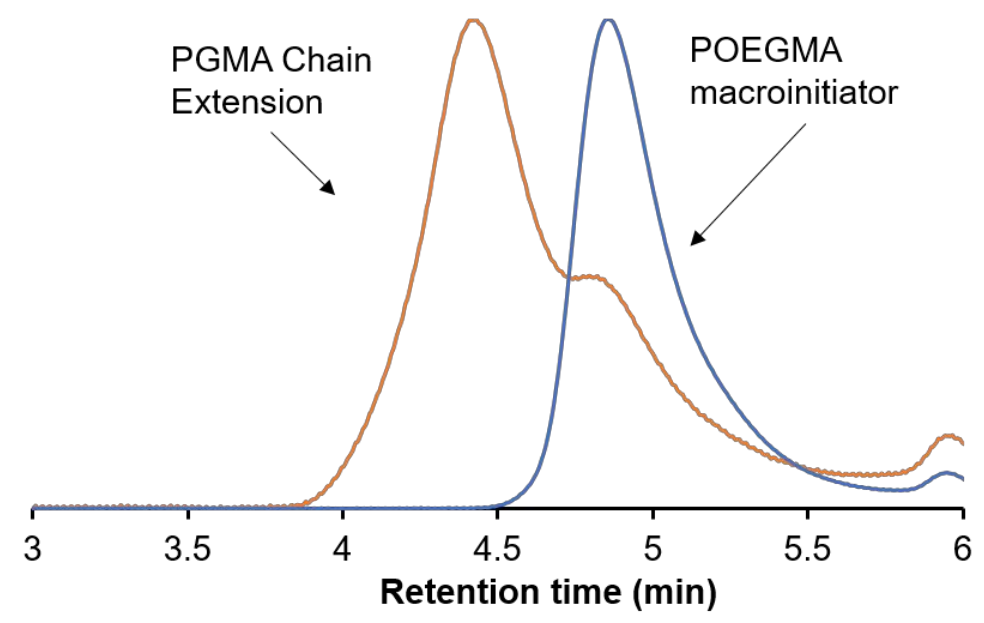

Figure S4. GPC chromatogram of attempted one-pot PhotoATR-PISA utilizing $\left[\mathrm{CuBr}_{2}\right]=360$ ppm (entry 4 in Table S1)

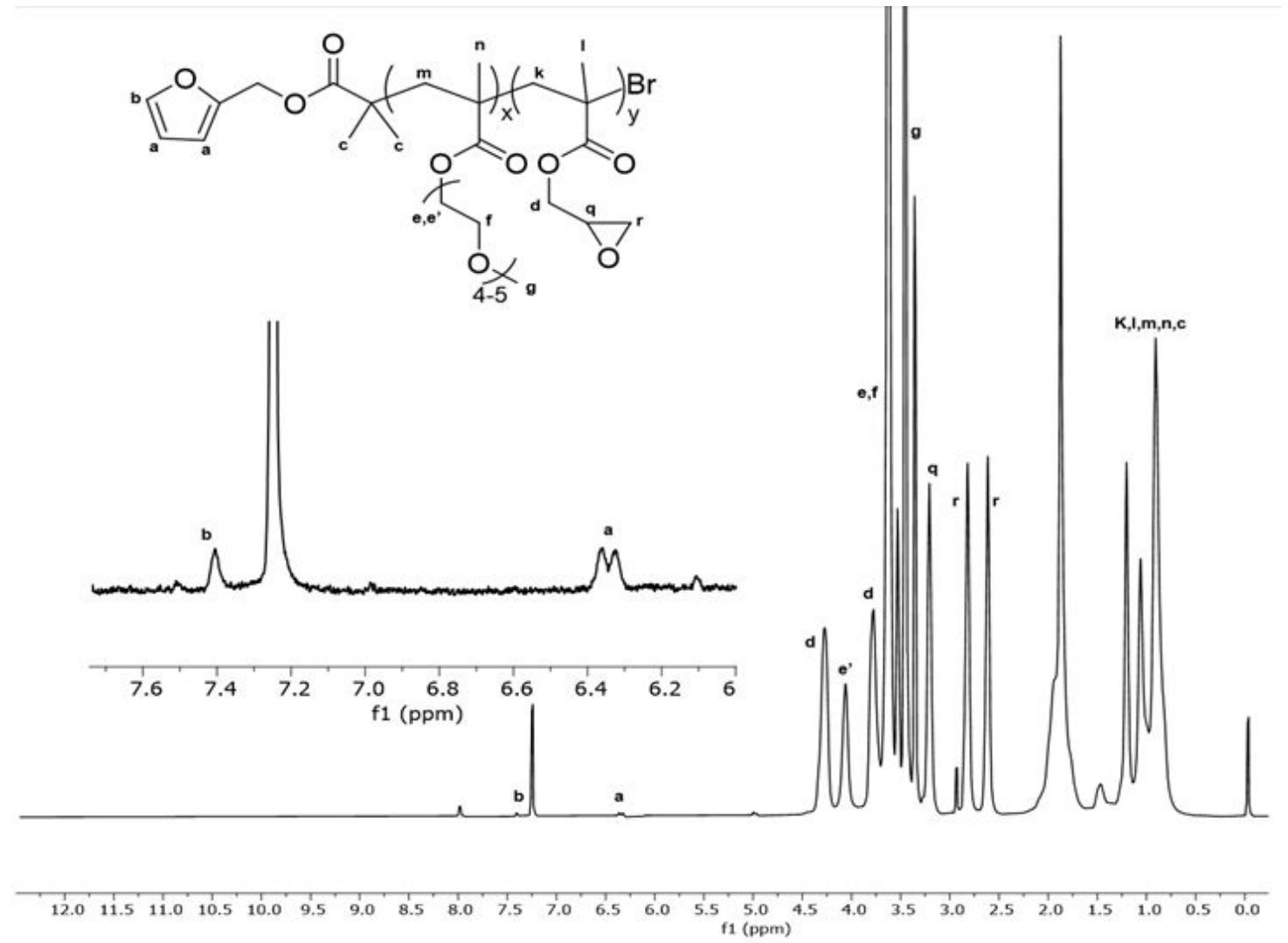


Figure S5. HNMR of the POEGMA-grad-PGMA copolymer for PISA4 (target DP(PGMA) = 86;

$\mathrm{SC} \%=32 \%)$
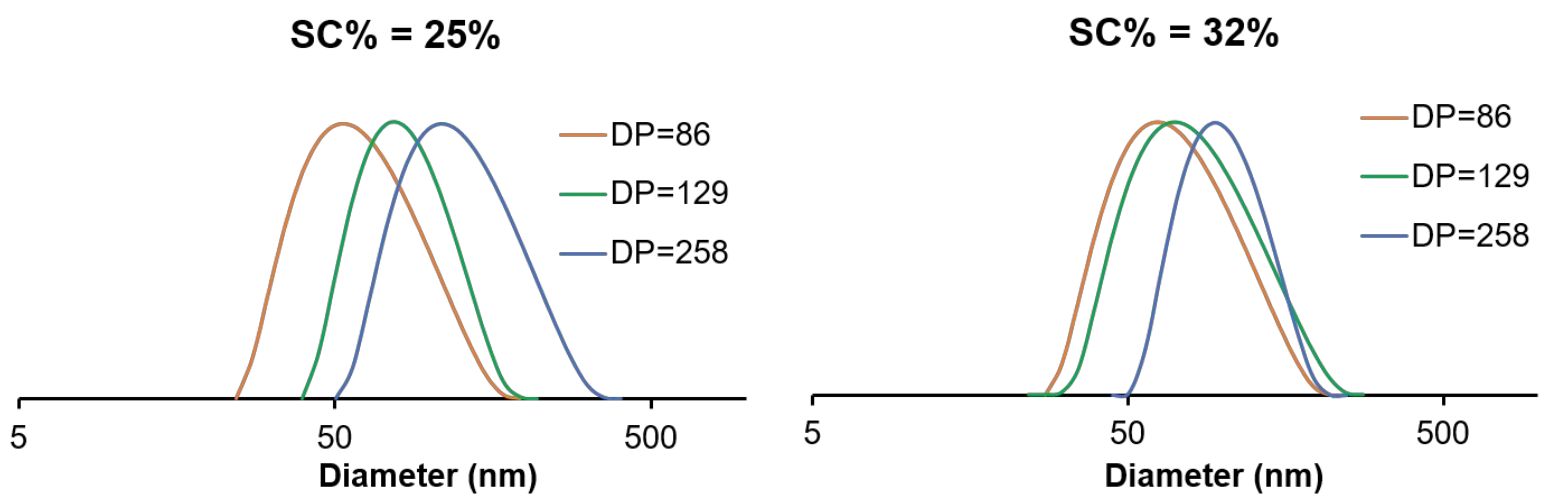

Figure S6. DLS of PISA1-6 in MeOH diluted to $0.5 \mathrm{mg} / \mathrm{mL}$ for analysis.

Table S1. Final PhotoATR-PISA GPC characterization and NP diameters as measured by AFM and DLS for DP(PGMA) $=86,129$ and 258 and two different $\mathrm{SC} \%=25$ (PISA1, 3 and 5, respectively) and 32\% (PISA2, 4 and 6, respectively).

\begin{tabular}{|c|c|c|c|c|c|c|}
\hline Entry & $\begin{array}{c}{ }^{2} \mathbf{D P} \\
\text { for the first block }\end{array}$ & $\begin{array}{c}{ }^{6} \mathbf{M}_{\mathbf{u}} \\
(\mathbf{k D a})\end{array}$ & $\begin{array}{c}{ }^{\mathrm{c}} \mathbf{M}_{\mathbf{u}, \text { th }} \\
(\mathbf{k D a})\end{array}$ & $\begin{array}{c}\text { dDiameter } \\
(\mathbf{n m})\end{array}$ & $\begin{array}{c}\text { 'Diameter } \\
(\mathbf{n m})\end{array}$ & $\begin{array}{c}\text { eS.D } \\
(\mathbf{n m})\end{array}$ \\
\hline PISA1 & 40 & 31.9 & 24.4 & 54 & 53 & 7.5 \\
\hline PISA2 & 37 & 24.7 & 23.4 & 59 & 116 & 57.2 \\
\hline PISA3 & 36 & 32.9 & 29.2 & 102 & 123 & 23.8 \\
\hline PISA4 & 33 & 28.8 & 28.2 & 82 & 103 & 31.9 \\
\hline PISA5 & 32 & 46.7 & 46.3 & 142 & 147 & 42.6 \\
\hline PISA6 & 40 & 69.4 & 49.0 & 115 & 104 & 27.0 \\
\hline
\end{tabular}

a Calculated by the $1 H$ NMR on the basis of considering the integration for the methylene protons (-CH2CH2OCO-) adjacent to the ester group in POEGMA at 4.0 ppm according to Eq S1: DPIst block $=\frac{\text { I at } 4 \mathrm{ppm}}{\text { I at } 6.3 \mathrm{ppm}}$.

b Calculated by the $1 H$ NMR on the basis of the Eq.S2. Eq. S2: DPIst block $\times 300+\frac{I \text { at } 2.7 \mathrm{ppm}}{I \text { at } 6.3 \mathrm{ppm}} \times 2 \times 142.15+246.106$.

c determined according to the Eq. S3. Eq. S3: Mn,th=300 $\times$ DPIst block+142.15 $\times$ targeted DP for the second block $\times$ conversion.

d Determined by DLS analysis. e Determined by AFM analysis (calculated by measuring at least 10 NPs diameters) 


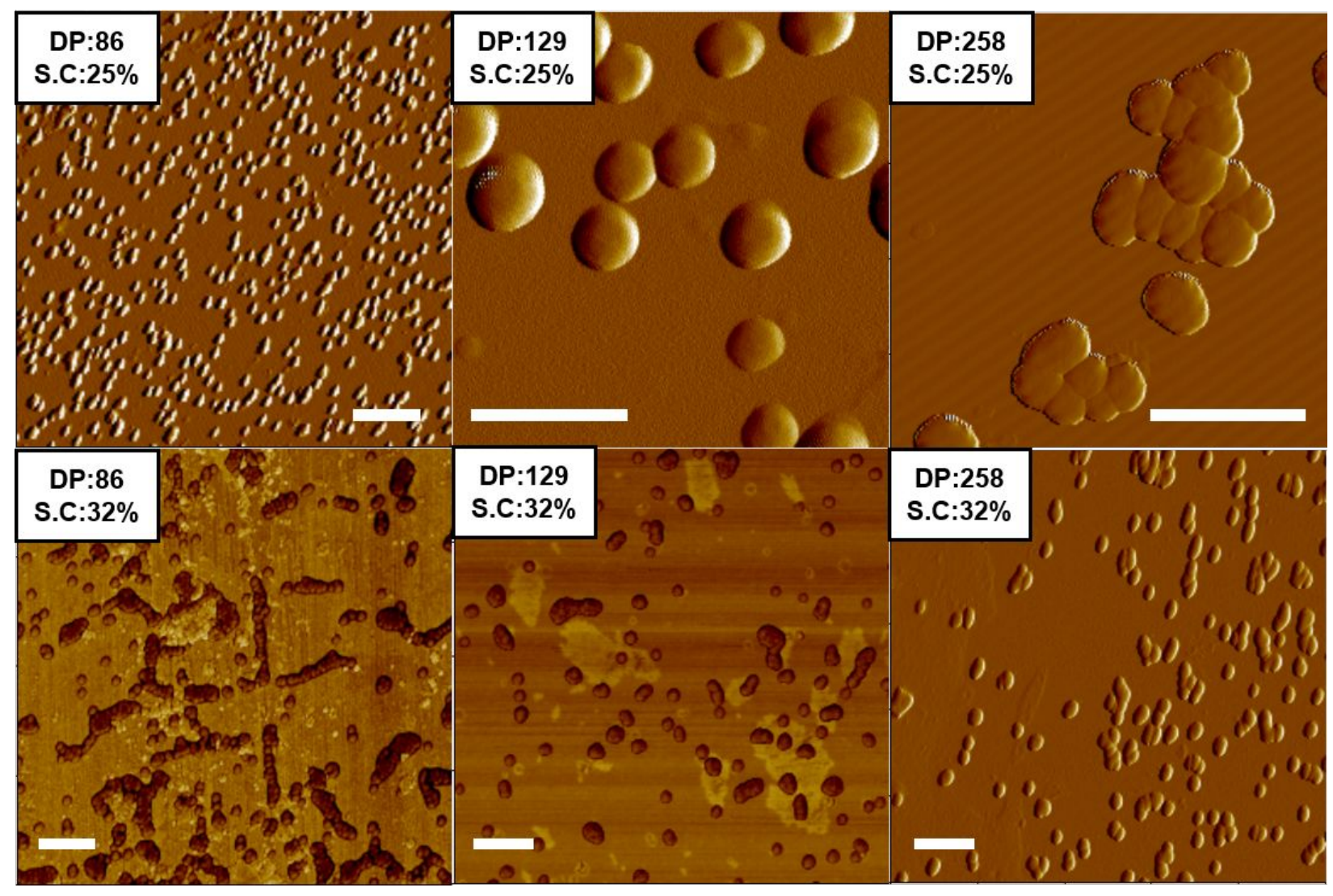

Figure S7. AFM phase micrographs for PISA1-6 (scale bars $=500 \mathrm{~nm})$.

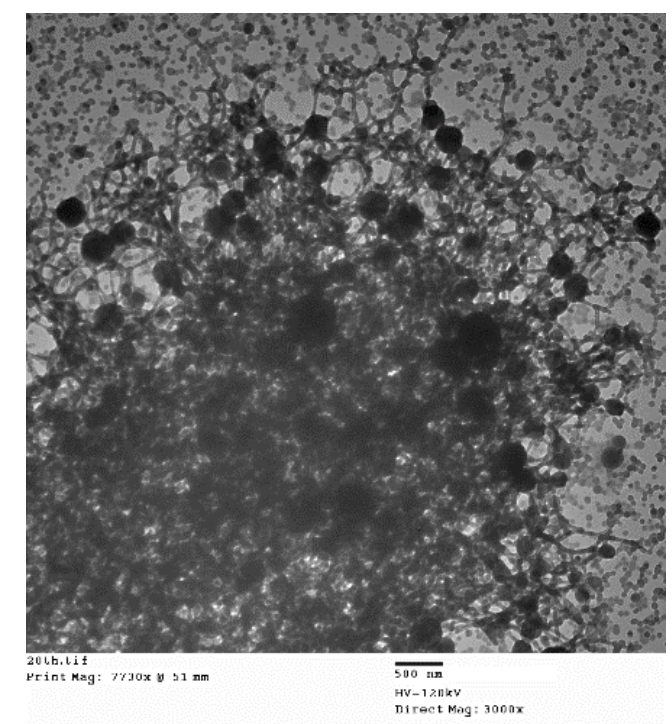

Figure S8. Low magnification image of tangled worm-like micelle networks that form upon thermal annealing of PISA5 samples at $65^{\circ} \mathrm{C}$ for $9 \mathrm{~h}$. 


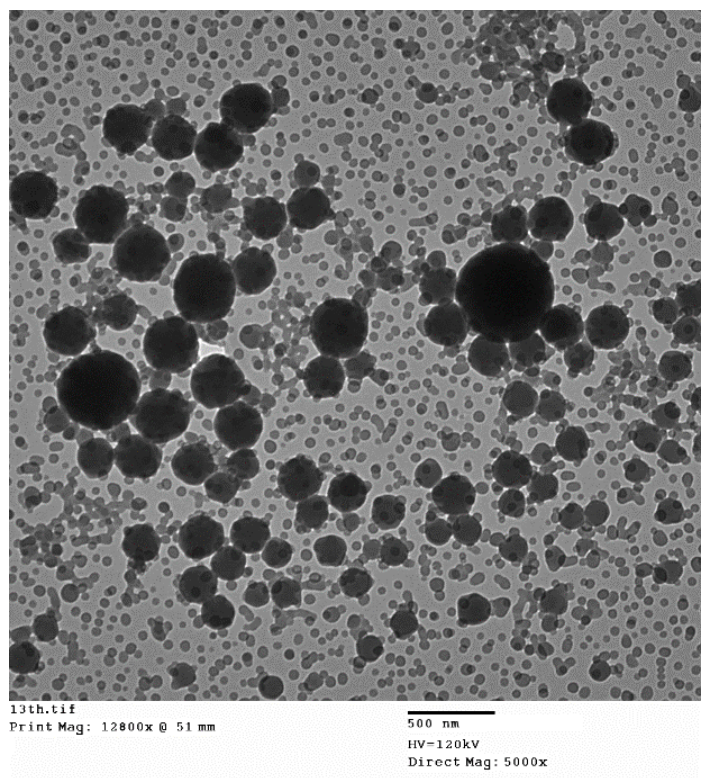

Figure S9. TEM micrograph of remaining dispersed nanostructures for PISA5 after $21 \mathrm{~h}$ of thermal annealing at $65^{\circ} \mathrm{C}$ showing mixtures of compound and individual nanospheres.

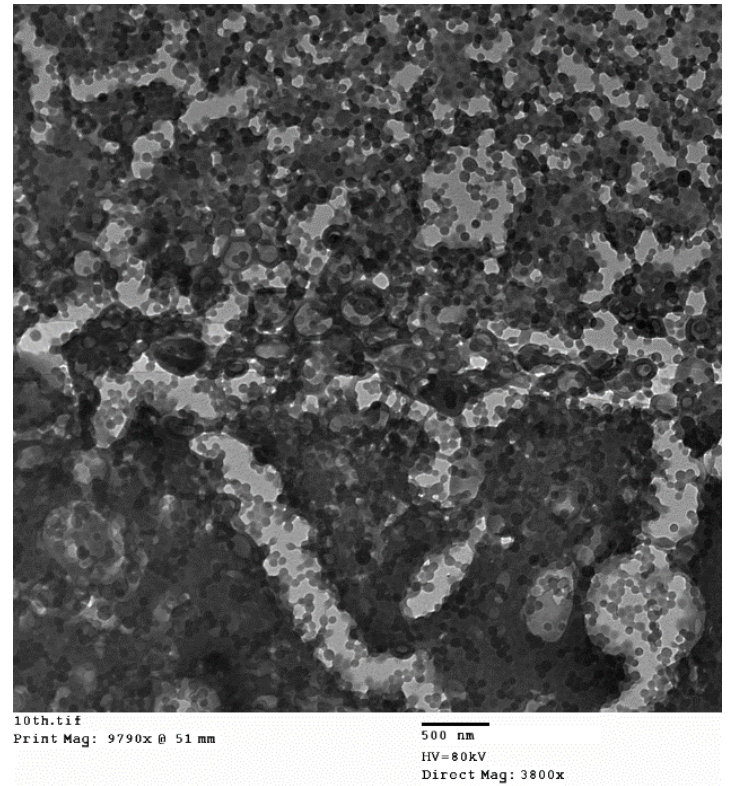

Figure S10. TEM image of mixed morphology nanostructures observed after thermal annealing of PISA6 for $35 \mathrm{~h}$ at $65^{\circ} \mathrm{C}$ showing what appears to be mixtures of vesicles, beaded worms and bilayer nanosheets. 


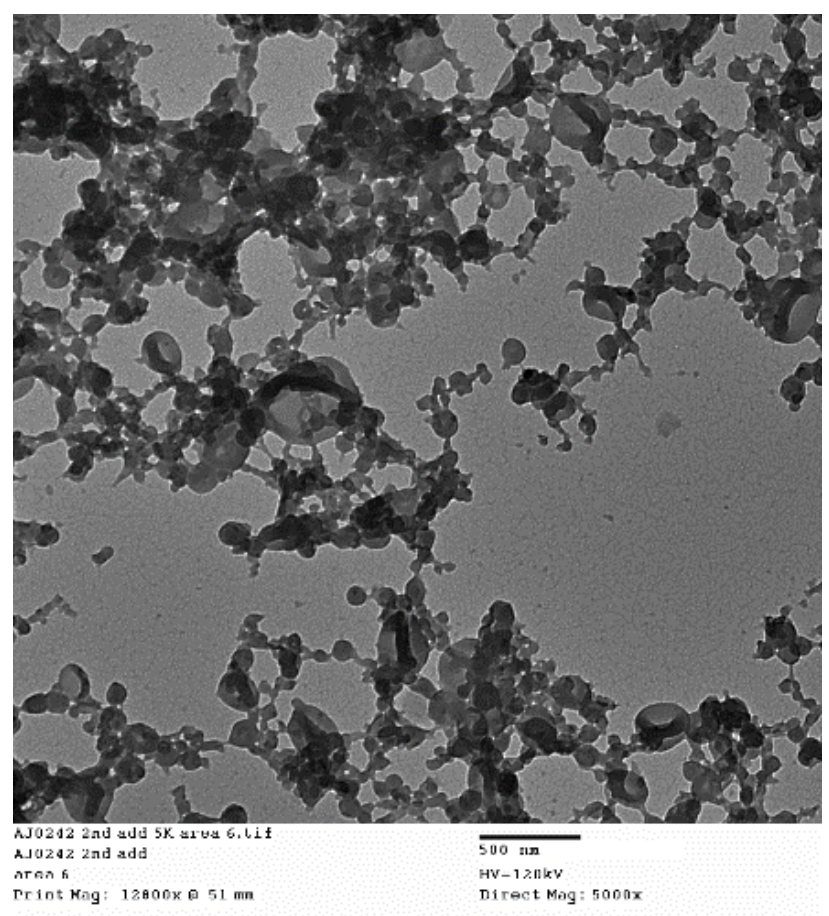

Figure S11. Lower magnification TEM image of vesicle nanostructures formed after the second chain extension (target $\mathrm{DP}_{\mathrm{PGMA}}=86$ per monomer addition).

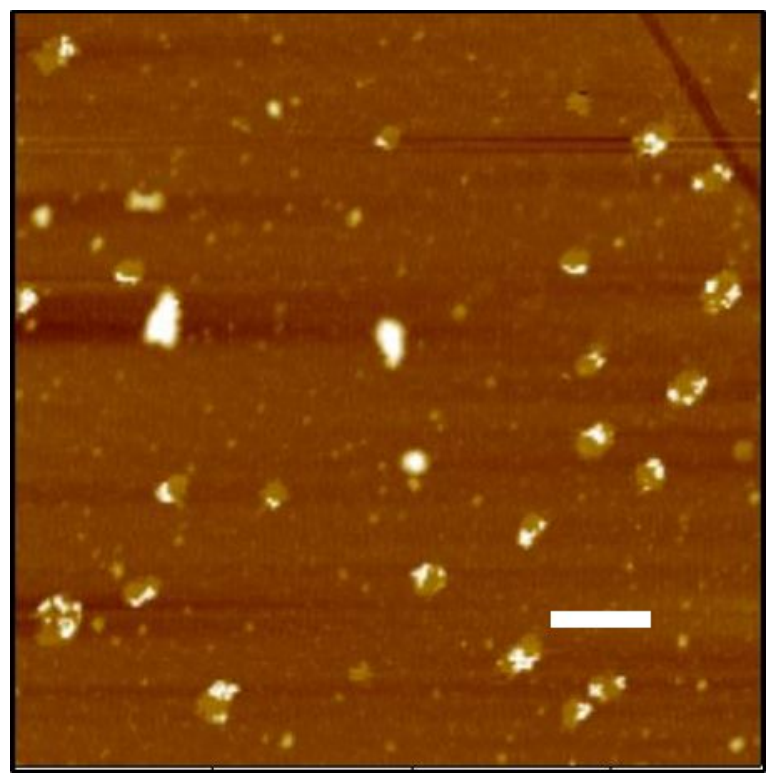


Figure S12. AFM image of collapsed vesicle nanostructures formed following the second chain extension for one-pot PhotoATR-PISA with target $\mathrm{DP}_{\mathrm{PGMA}}=86$ per monomer addition (white scale bar $=1.0 \mu \mathrm{m})$

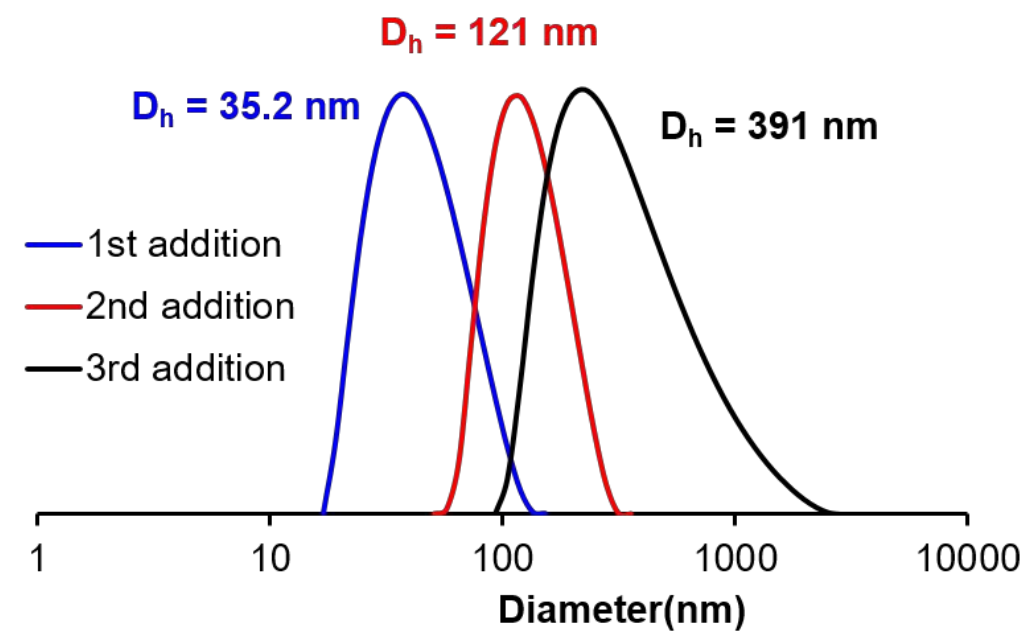

Figure S13. DLS analysis for the three sequential chain extensions of PGMA from POEGMA macroinitiators.
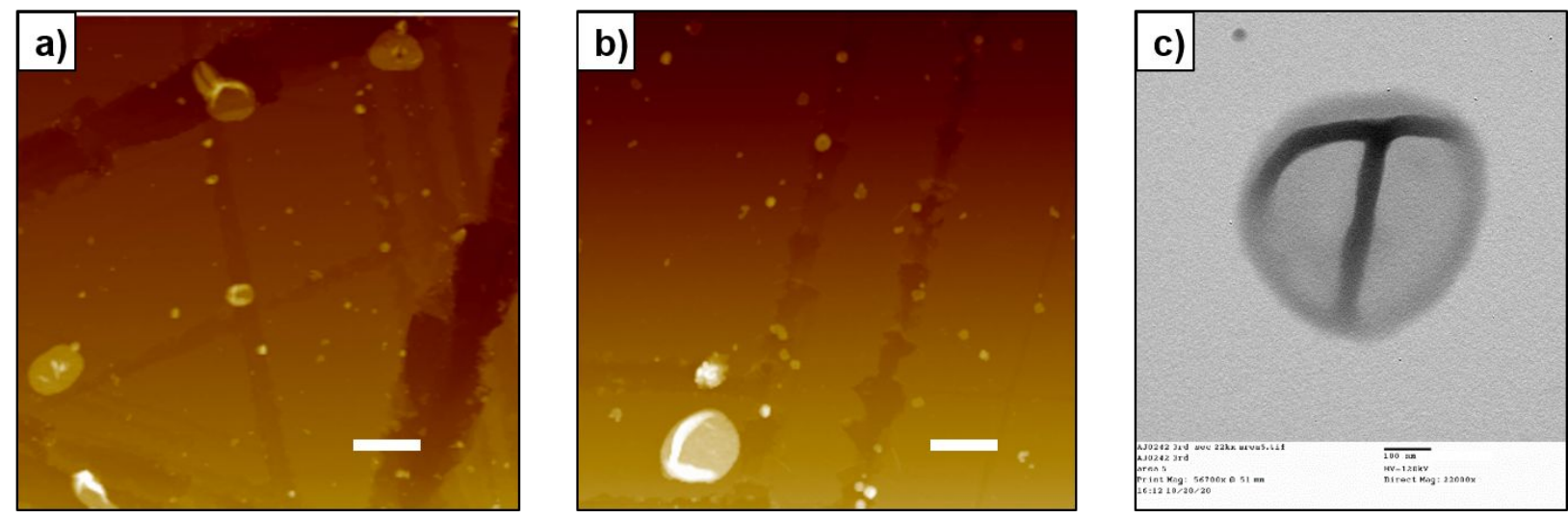

Figure S14. AFM (a,b) and TEM (c) images of dispersed vesicle nanostructures formed following the third chain extension for one-pot PhotoATR-PISA with target $\mathrm{DP}_{\mathrm{PGMA}}=86$ per monomer addition. 


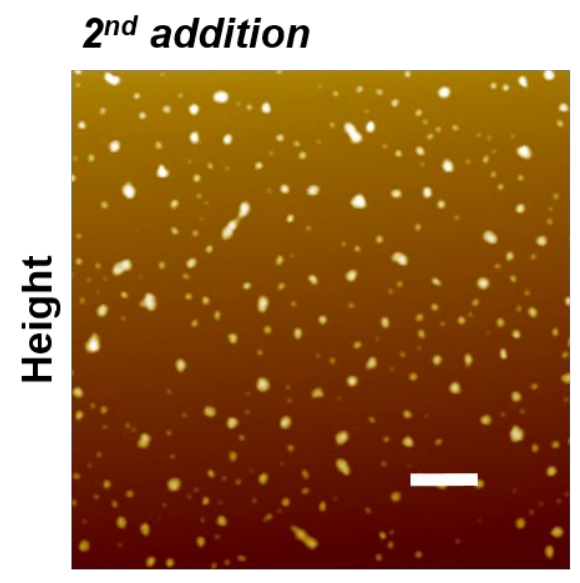

\section{$3^{\text {rd }}$ addition}
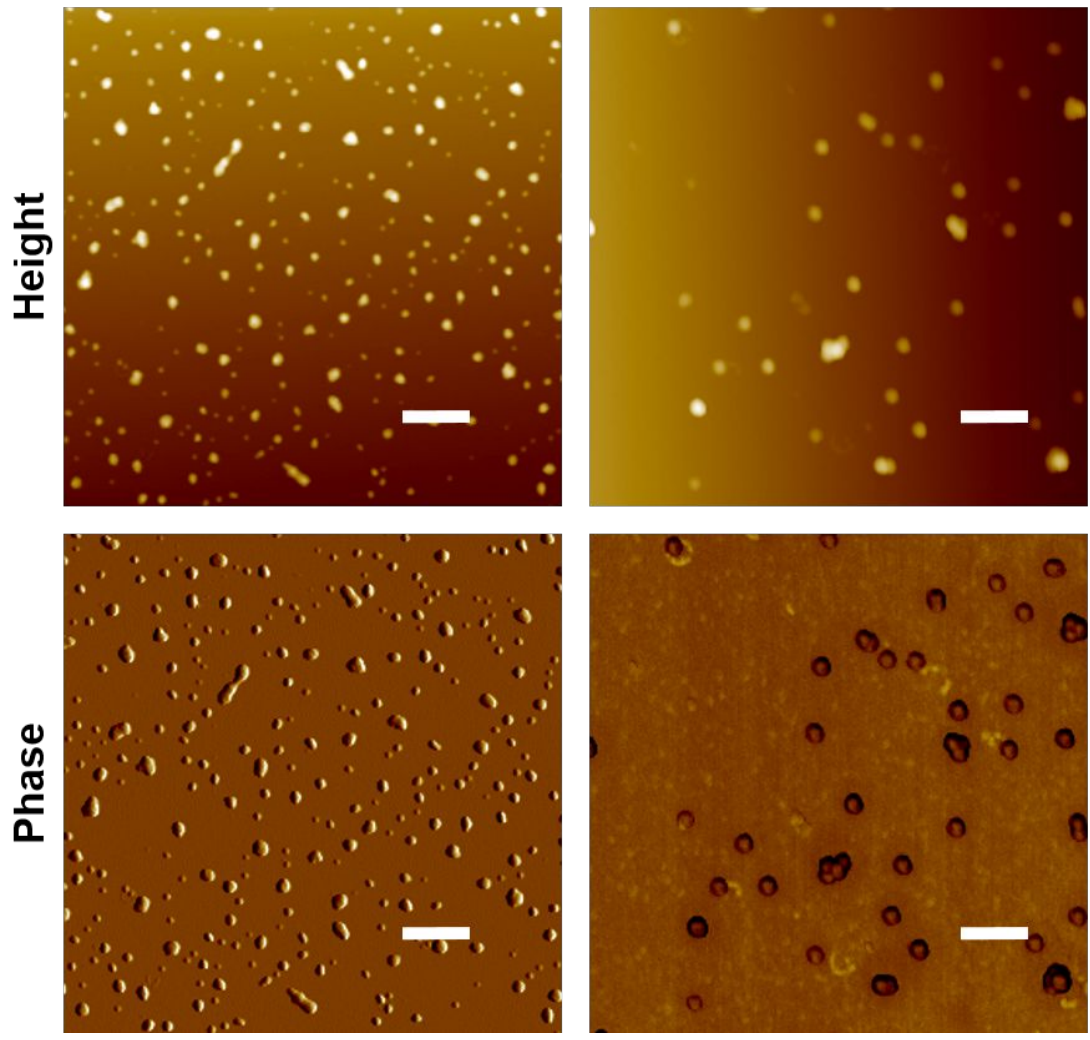

$4^{\text {th }}$ addition
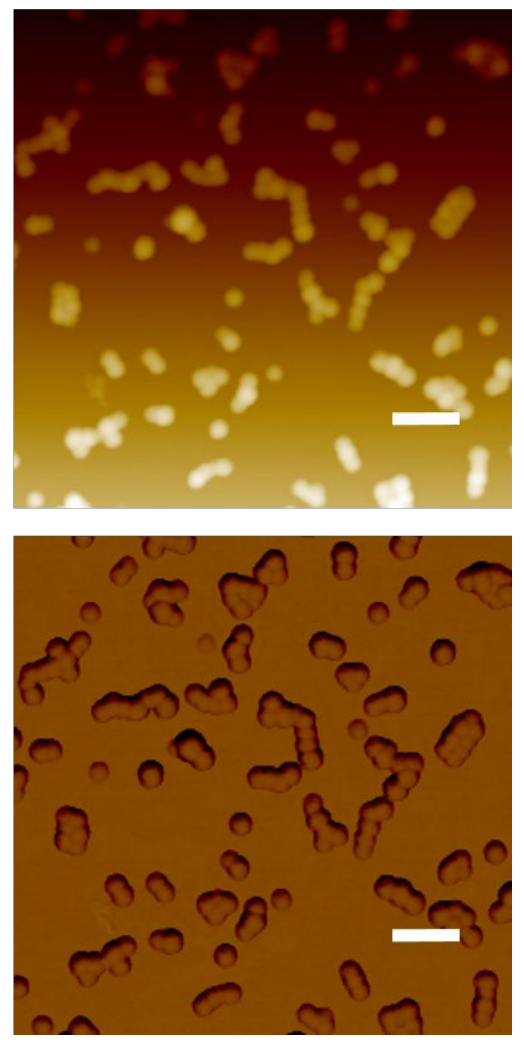

Figure S15. AFM images of one-pot PhotoATR-PISA after the $2^{\text {nd }}, 3^{\text {rd }}$ and $4^{\text {th }}$ chain extension showing transitions from spheres to beaded worms (target $\mathrm{DP}_{\mathrm{PGMA}}=43$ per monomer addition) 


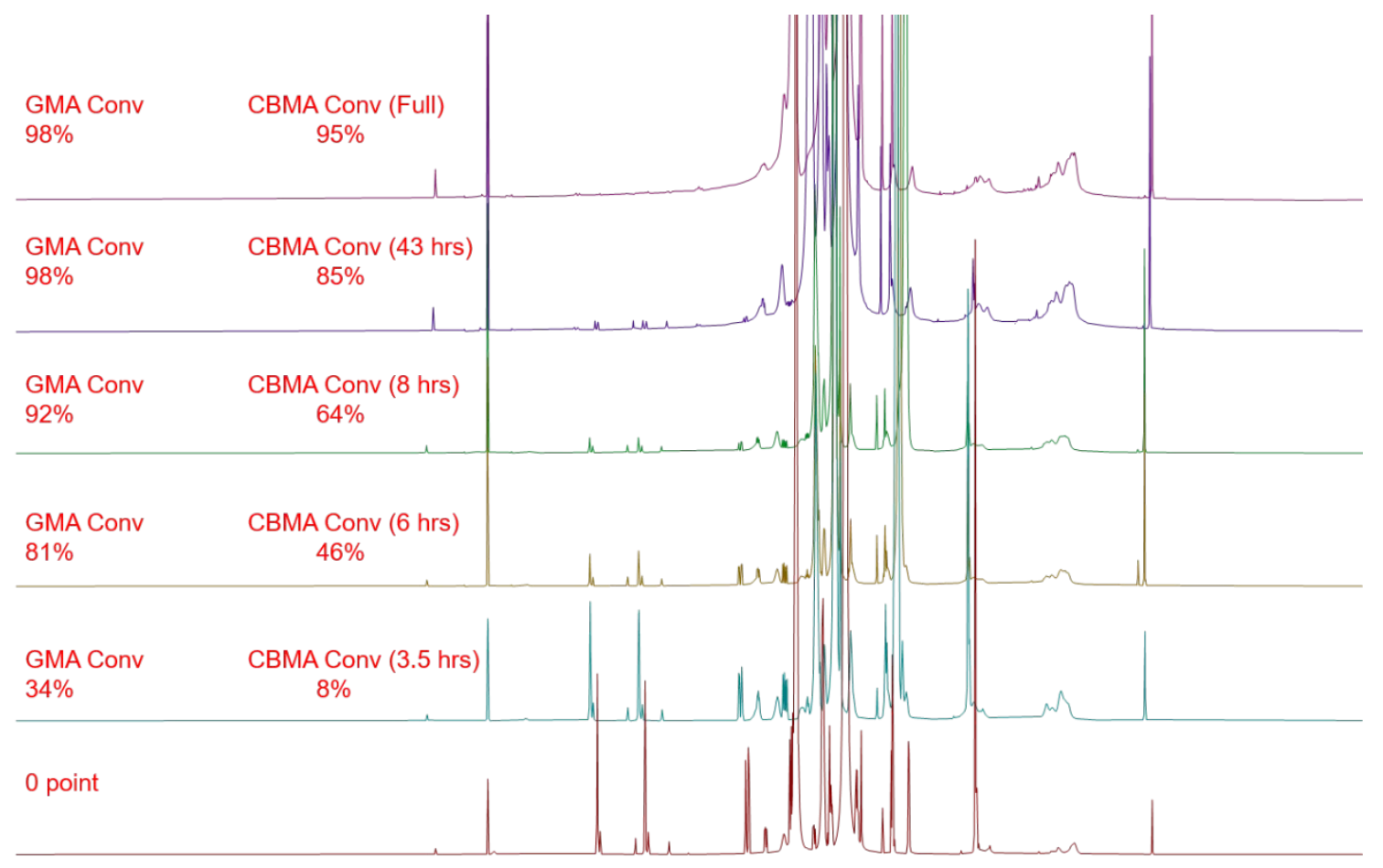

$\begin{array}{lllllllllllllllllllllllllllllllll}12.0 & 11.5 & 11.0 & 10.5 & 10.0 & 9.5 & 9.0 & 8.5 & 8.0 & 7.5 & 7.0 & 6.5 & 6.0 & 5.5 & 5.0 & 4.5 & 4.0 & 3.5 & 3.0 & 2.5 & 2.0 & 1.5 & 1.0 & 0.5 & 0.0 & -0.5 & -1.0 & -1.5 & -2.0\end{array}$

Figure S16. ${ }^{1} \mathrm{H}$ NMR analysis of one-pot CCL protocol utilizing CBMA crosslinkers in PhotoATR-PISA showing slow consumption of CBMA compared to GMA.
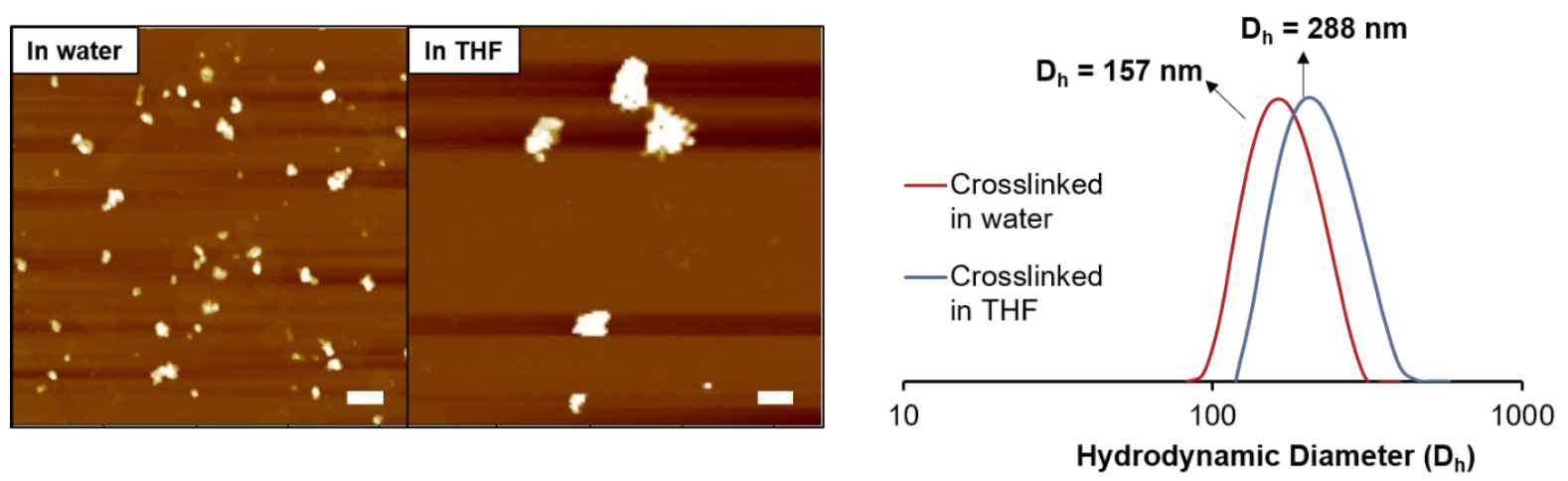

Figure S17. AFM images (scale bars $=400 \mathrm{~nm}$ ) and DLS of PhotoATR-PISA CCL NPs in water (left; red) and THF (right; blue). 


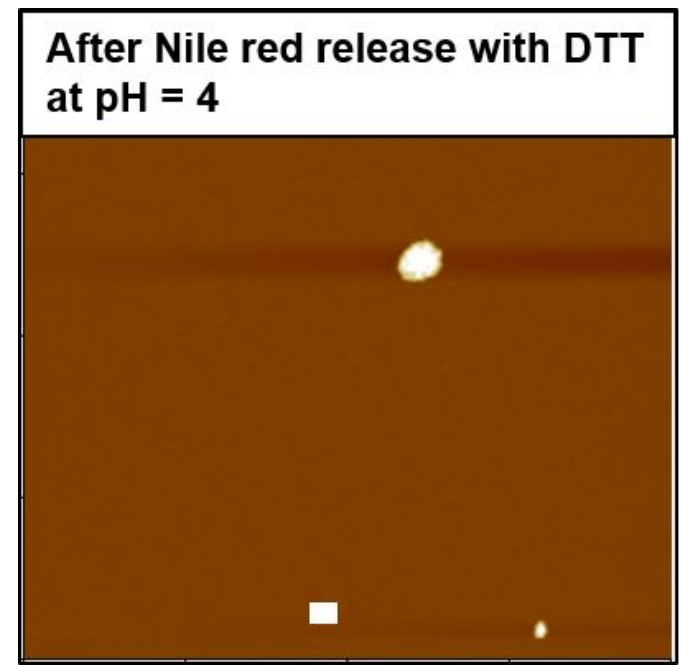

Figure S18. AFM image of CCL NPs after exposure to DTT in water $(\mathrm{pH}=4)$ for $120 \mathrm{~h}$ showing only one intact NP (scale bar $=400 \mathrm{~nm})$.

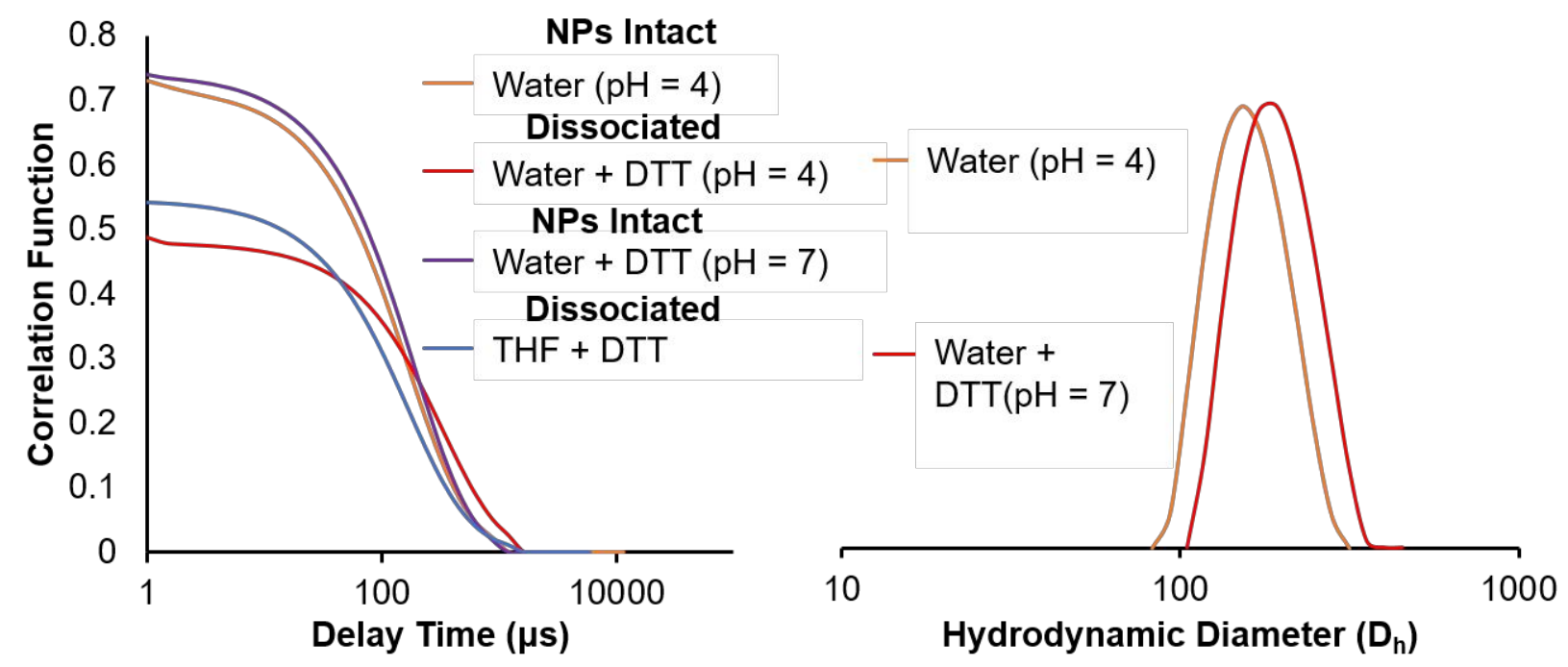

Figure S19. DLS correlation function for intact NPs in water at $\mathrm{pH}=4$ without DTT and water + DTT at $\mathrm{pH}=7$ as well as dissociated NPs in water + DTT at $\mathrm{pH}=4$ and in THF + DTT. 


\section{References}

1. Chen, M.; Li, J.-W.; Zhang, W.-J.; Hong, C.-Y.; Pan, C.-Y. pH- and Reductant-Responsive Polymeric Vesicles with Robust Membrane-Cross-Linked Structures: In Situ Cross-Linking in Polymerization-Induced Self-Assembly. Macromolecules 2019, 52 (3), 1140-1149. 